\title{
Lanczos-Lovelock and $f(R)$ Gravity from Clifford Space Geometry
}

\author{
Carlos Castro \\ Center for Theoretical Studies of Physical Systems \\ Clark Atlanta University, Atlanta, Georgia. 30314, perelmanc@hotmail.com
}

May 2012

\begin{abstract}
A rigorous construction of Clifford-space Gravity is presented which is compatible with the Clifford algebraic structure and permits the derivation of the generalized connections in Clifford spaces ( $C$-space) in terms of derivatives of the $C$-space metric. We continue by arguing how Lanczos-Lovelock higher curvature gravity can be embedded into gravity in Clifford spaces and suggest how this might also occur for extended gravitational theories based on $f(R), f\left(R_{\mu \nu}\right), \ldots$ actions, for polynomial-valued functions. Black-strings and black-brane metric solutions in higher dimensions $D>4$ play an important role in finding specific examples.
\end{abstract}

\section{The construction of Clifford-space Gravity}

At the beginning of this section we follow closely the work in [1] and then we depart from it by constructing Clifford space ( $C$-space) gravity without making any a priori assumptions on the $C$-space connections.

Let the vector fields $\gamma_{\mu}, \mu=1,2, \ldots, n$ be a coordinate basis in $V_{n}$ satisfying the Clifford algebra relation

$$
\gamma_{\mu} \cdot \gamma_{\nu} \equiv \frac{1}{2}\left(\gamma_{\mu} \gamma_{\nu}+\gamma_{\nu} \gamma_{\mu}\right)=g_{\mu \nu}
$$

where $g_{\mu \nu}$ is the metric of $V_{n}$. In curved space $\gamma_{\mu}$ and $g_{\mu \nu}$ cannot be constant but necessarily depend on position $x^{\mu}$. An arbitrary vector is a linear superposition [2]

$$
a=a^{\mu} \gamma_{\mu}
$$

where the components $a^{\mu}$ are scalars from the geometric point of view, whilst $\gamma_{\mu}$ are vectors. 
Besides the basis $\left\{\gamma_{\mu}\right\}$ we can introduce the reciprocal basis ${ }^{1}\left\{\gamma^{\mu}\right\}$ satisfying

$$
\gamma^{\mu} \cdot \gamma^{\nu} \equiv \frac{1}{2}\left(\gamma^{\mu} \gamma^{\nu}+\gamma^{\nu} \gamma^{\mu}\right)=g^{\mu \nu}
$$

where $g^{\mu \nu}$ is the covariant metric tensor such that $g^{\mu \alpha} g_{\alpha \nu}=\delta^{\mu}{ }_{\nu}, \gamma^{\mu} \gamma_{\nu}+\gamma_{\nu} \gamma^{\mu}=2 \delta^{\mu}{ }_{\nu}$ and $\gamma^{\mu}=g^{\mu \nu} \gamma_{\nu}$

Following ref.[2] (see also [3]) we consider the vector derivative or gradient defined according to

$$
\partial \equiv \gamma^{\mu} \partial_{\mu}
$$

where $\partial_{\mu}$ is an operator whose action depends on the quantity it acts on. Applying the vector derivative $\partial$ on a scalar field $\phi$ we have

$$
\partial \phi=\gamma^{\mu} \partial_{\mu} \phi
$$

where $\partial_{\mu} \phi \equiv\left(\partial / \partial x^{\mu}\right) \phi$ coincides with the partial derivative of $\phi$.

But if we apply it on a vector field $a$ we have

$$
\partial a=\gamma^{\mu} \partial_{\mu}\left(a^{\nu} \gamma_{\nu}\right)=\gamma^{\mu}\left(\partial_{\mu} a^{\nu} \gamma_{\nu}+a^{\nu} \partial_{\mu} \gamma_{\nu}\right)
$$

In general $\gamma_{\nu}$ is not constant; it satisfies the relation [2], [3]

$$
\partial_{\mu} \gamma_{\nu}=\Gamma_{\mu \nu}^{\alpha} \gamma_{\alpha}
$$

where $\Gamma_{\mu \nu}^{\alpha}$ is the connection. Similarly, for $\gamma^{\nu}=g^{\nu \alpha} \gamma_{\alpha}$ we have

$$
\partial_{\mu} \gamma^{\nu}=\Gamma_{\mu}^{\nu}{ }_{\alpha} \gamma^{\alpha}=-\Gamma_{\mu \alpha}^{\nu} \gamma^{\alpha}
$$

For further references on Clifford algebras see [4], [5].

The non commuting operator $\partial_{\mu}$ so defined determines the parallel transport of a basis vector $\gamma^{\nu}$. Instead of the symbol $\partial_{\mu}$ Hestenes uses $\square_{\mu}$, whilst Misner, Thorne and Wheeler, use $\nabla_{\mu}$ and call it "covariant derivative". In modern, mathematically oriented literature more explicit notation such as $\mathrm{D}_{\gamma_{\mu}}$ or $\nabla_{\gamma_{\mu}}$ is used. However, such a notation, although mathematically very relevant, would not be very practical in long computations. We find it very convenient to keep the symbol $\partial_{\mu}$ for components of the geometric operator $\partial=\gamma^{\mu} \partial_{\mu}$. When acting on a scalar field the derivative $\partial_{\mu}$ happens to be commuting and thus behaves as the ordinary partial derivative. When acting on a vector field, $\partial_{\mu}$ is a non commuting operator. In this respect, there can be no confusion with partial derivative, because the latter normally acts on scalar fields, and in such a case partial derivative and $\partial_{\mu}$ are one and the same thing. However, when acting on a vector field, the derivative $\partial_{\mu}$ is non commuting. Our operator $\partial_{\mu}$ when acting on $\gamma_{\mu}$ or $\gamma^{\mu}$ should be distinguished from the ordinary - commuting - partial derivative, let be denoted $\gamma^{\nu}{ }_{, \mu}$, usually used in the literature on the Dirac equation in curved spacetime. The latter derivative is not used in the present paper, so there should be no confusion.

\footnotetext{
${ }^{1}$ In Appendix A of the Hesteness book [2] the frame $\left\{\gamma^{\mu}\right\}$ is called dual frame because the duality operation is used in constructing it.
} 
Using (7), eq.(6) becomes

$$
\partial a=\gamma^{\mu} \gamma_{\nu}\left(\partial_{\mu} a^{\nu}+\Gamma_{\mu \alpha}^{\nu} a^{\alpha}\right) \equiv \gamma^{\mu} \gamma_{\nu} \mathrm{D}_{\mu} a^{\nu}=\gamma^{\mu} \gamma^{\nu} \mathrm{D}_{\mu} a_{\nu}
$$

where $\mathrm{D}_{\mu}$ is the covariant derivative of tensor analysis..

Let us now consider $C$-space and very briefly review the procedure of [1]. A basis in $C$-space is given by

$$
E_{A}=\left\{\gamma, \gamma_{\mu}, \gamma_{\mu} \wedge \gamma_{\nu}, \gamma_{\mu} \wedge \gamma_{\nu} \wedge \gamma_{\rho}, \ldots\right\}
$$

where $\gamma$ is the unit element of the Clifford algebra that we label as $\mathbf{1}$ from now on. In an $r$-vector $\gamma_{\mu_{1}} \wedge \gamma_{\mu_{2}} \wedge \ldots \wedge \gamma_{\mu_{r}}$ we take the indices so that $\mu_{1}<\mu_{2}<\ldots<\mu_{r}$. An element of $C$-space is a Clifford number, called also Polyvector or Clifford aggregate which we now write in the form

$$
X=X^{A} E_{A}=s \mathbf{1}+x^{\mu} \gamma_{\mu}+x^{\mu \nu} \gamma_{\mu} \wedge \gamma_{\nu}+\ldots
$$

A $C$-space is parametrized not only by 1-vector coordinates $x^{\mu}$ but also by the 2-vector coordinates $x^{\mu \nu}, 3$-vector coordinates $x^{\mu \nu \alpha}$, etc., called also holographic coordinates, since they describe the holographic projections of 1-loops, 2-loops, 3-loops, etc., onto the coordinate planes. By $p$-loop we mean a closed p-brane; in particular, a 1-loop is closed string. In order to avoid using the powers of the Planck scale length parameter $L_{p}$ in the expansion of the poly-vector $X$ we can set set to unity to simplify matters.

In a flat $C$-space the basis vectors $E^{A}$ are constants. In a curved $C$-space this is no longer true. Each $E_{A}$ is a function of the $C$-space coordinates

$$
X^{A}=\left\{s, x^{\mu}, x^{\mu \nu}, \ldots\right\}
$$

which include scalar, vector, bivector,..., $r$-vector,.., coordinates. Now we define the connection $\tilde{\Gamma}_{A B}^{C}$ in $C$-space according to

$$
\partial_{A} E_{B}=\tilde{\Gamma}_{A B}^{C} E_{C}
$$

where $\partial_{A} \equiv \partial / \partial X^{A}$ is the derivative in $C$-space. This definition is analogous to the one in ordinary space. Let us therefore define the $C$-space curvature as

$$
\mathcal{R}_{A B C}{ }^{D}=\left(\left[\partial_{A}, \partial_{B}\right] E_{C}\right) * E^{D}
$$

which is a straightforward generalization of the ordinary relation in Riemannian geometry.

The 'star' means the scalar product between two polyvectors $A$ and $B$, defined as

$$
A * B=\langle A B\rangle_{S}
$$

where ' $S$ ' means 'the scalar part' of the geometric product $A B$.

In [1] we explored the above relation for curvature and showed how it was related to the curvature of the ordinary space. After making several assumptions we were able to demonstrate that the derivative with respect to the bivector coordinate $x^{\mu \nu}$ is equal to the commutator of the derivatives with respect to the vector coordinates $x^{\mu}$. This will not be the case in this work. 
Returning now to eq.(13), the differential of a $C$-space basis vector is given by

$$
\mathrm{d} E_{A}=\frac{\partial E_{A}}{\partial X^{B}} \mathrm{~d} X^{B}=\Gamma_{A B}^{C} E_{C} \mathrm{~d} X^{B}
$$

In particular, for $A=\mu$ and $E_{A}=\gamma_{\mu}$ we have

$$
\begin{aligned}
\mathrm{d} \gamma_{\mu}= & \frac{\partial \gamma_{\mu}}{\partial X^{\nu}} \mathrm{d} x^{\nu}+\frac{\partial \gamma_{\mu}}{\partial x^{\alpha \beta}} \mathrm{d} x^{\alpha \beta}+\ldots=\tilde{\Gamma}_{\nu \mu}^{A} E_{A} \mathrm{~d} x^{\nu}+\tilde{\Gamma}_{[\alpha \beta] \mu}^{A} E_{A} \mathrm{~d} x^{\alpha \beta}+\ldots \\
= & \left(\tilde{\Gamma}_{\nu \mu}^{\alpha} \gamma_{\alpha}+\tilde{\Gamma}_{\nu \mu}^{[\rho \sigma]} \gamma_{\rho} \wedge \gamma_{\sigma}+\ldots\right) \mathrm{d} x^{\nu} \\
& +\left(\tilde{\Gamma}_{[\alpha \beta] \mu}^{\rho} \gamma_{\rho}+\tilde{\Gamma}_{[\alpha \beta] \mu}^{[\rho \sigma]} \gamma_{\rho} \wedge \gamma_{\sigma}+\ldots\right) \mathrm{d} x^{\alpha \beta}+\ldots
\end{aligned}
$$

We see that the differential $\mathrm{d} \gamma_{\mu}$ is in general a polyvector, i.e., a Clifford aggregate. In eq.(17) we have used

$$
\begin{gathered}
\frac{\partial \gamma_{\mu}}{\partial x^{\nu}}=\tilde{\Gamma}_{\nu \mu}^{\alpha} \gamma_{\alpha}+\tilde{\Gamma}_{\nu \mu}^{[\rho \sigma]} \gamma_{\rho} \wedge \gamma_{\sigma}+\ldots \\
\frac{\partial \gamma_{\mu}}{\partial x^{\alpha \beta}}=\tilde{\Gamma}_{[\alpha \beta] \mu}^{\rho} \gamma_{\rho}+\tilde{\Gamma}_{[\alpha \beta] \mu}^{[\rho \sigma]} \gamma_{\rho} \wedge \gamma_{\sigma}+\ldots
\end{gathered}
$$

In this work we will not impose, by hand, any conditions and we have now that

$$
\partial_{\mu \nu} \neq\left[\partial_{\mu}, \partial_{\nu}\right], \quad \Gamma_{[\alpha \beta]_{\mu}}^{\rho} \neq R_{\alpha \beta \mu}{ }^{\rho}, \quad \partial_{\mu_{1} \mu_{2}} g^{\rho \tau} \neq 0, \partial_{\mu_{1} \mu_{2}} R_{\alpha \beta \mu}{ }^{\rho} \neq 0
$$

so the $C$-space scalar curvature $\mathbf{R}$ does not longer decompose as in [1]

$$
\mathbf{R}=R+\alpha_{1} R^{2}+\alpha_{2} R_{\mu \nu} R^{\mu \nu}+\ldots
$$

but rather it bears a closer relationship to Lanczos-Lovelock gravity as we shall see in the next section. We will derive the $C$-space connections from the Clifford algebraic structure, and without any a priori assumptions, as follows.

The Clifford scalar component $\mathbf{s}$ of the poly-vector $\mathbf{X}=X_{A} E^{A}$ will be labeled with the index $\mathbf{0}$ from now on and must not be confused with the temporal component of the vector $x^{\mu}$. Let us begin by writing

$$
\begin{aligned}
& \partial_{0}\left\{\gamma^{\mu}, \gamma^{\nu}\right\}=2 \partial_{0} g^{\mu \nu} \Rightarrow \\
& \Gamma_{\mathbf{0} \sigma}^{\mu} \gamma^{\sigma} \gamma^{\nu}+\gamma^{\mu} \Gamma_{\mathbf{0} \sigma}^{\nu} \gamma^{\sigma}+\Gamma_{\mathbf{0} \sigma}^{\nu} \gamma^{\sigma} \gamma^{\mu}+\gamma^{\nu} \Gamma_{\mathbf{0} \sigma}^{\mu} \gamma^{\sigma}= \\
& 4 \Gamma_{\mathbf{0}}^{\mu \nu}=2 \partial_{\mathbf{0}} g^{\mu \nu} \Rightarrow \Gamma_{\mathbf{0}}^{\mu \nu}=\frac{1}{2} \partial_{\mathbf{0}} g^{\mu \nu}
\end{aligned}
$$

Eq- (22) is obtained after using the relations

$$
\gamma^{\sigma} \gamma^{\nu}=\frac{1}{2}\left\{\gamma^{\sigma}, \gamma^{\nu}\right\}+\frac{1}{2}\left[\gamma^{\sigma}, \gamma^{\nu}\right]=g^{\sigma \nu}+\gamma^{\sigma \nu}
$$

with symmetric $g^{\sigma \nu}$, antisymmetric $\gamma^{\sigma \nu}=-\gamma^{\nu \sigma}$ and symmetric $\Gamma_{\mathbf{0}}^{\mu \nu}=\Gamma_{\mathbf{0}}^{\nu \mu}$. Taking derivatives with respect to $x^{\rho}$ gives

$$
\partial_{\rho}\left\{\gamma^{\mu}, \gamma^{\nu}\right\}=2 \partial_{\rho} g^{\mu \nu} \Rightarrow
$$




$$
\begin{gathered}
\Gamma_{\rho \sigma}^{\mu} \gamma^{\sigma} \gamma^{\nu}+\gamma^{\mu} \Gamma_{\rho \sigma}^{\nu} \gamma^{\sigma}+\Gamma_{\rho \sigma}^{\nu} \gamma^{\sigma} \gamma^{\mu}+\gamma^{\nu} \Gamma_{\rho \sigma}^{\mu} \gamma^{\sigma}= \\
4 \Gamma_{\rho}^{\mu \nu}=2 \partial_{\rho} g^{\mu \nu} \Rightarrow \Gamma_{\rho}^{\mu \nu}=\frac{1}{2} \partial_{\rho} g^{\mu \nu}
\end{gathered}
$$

Taking derivatives of the commutator

$$
\partial_{0}\left[\gamma^{\mu}, \gamma^{\nu}\right]=2 \partial_{0} \gamma^{\mu \nu}, \quad \partial_{\rho}\left[\gamma^{\mu}, \gamma^{\nu}\right]=2 \partial_{\rho} \gamma^{\mu \nu}
$$

gives the following relations

$$
\begin{aligned}
& \Gamma_{\mathbf{0} \sigma}^{\mu} \gamma^{\sigma \nu}-\Gamma_{\mathbf{0} \sigma}^{\nu} \gamma^{\sigma \mu}=\Gamma_{\mathbf{0} \tau_{1} \tau_{2}}^{[\mu \nu]} \gamma^{\tau_{1} \tau_{2}} \\
& \Gamma_{\rho \sigma}^{\mu} \gamma^{\sigma \nu}-\Gamma_{\rho \sigma}^{\nu} \gamma^{\sigma \mu}=\Gamma_{\rho \tau_{1} \tau_{2}}^{[\mu \nu]} \gamma^{\tau_{1} \tau_{2}}
\end{aligned}
$$

after having

$$
\partial_{\mathbf{0}} \gamma^{\mu \nu}=\Gamma_{\mathbf{0} \tau_{1} \tau_{2}}^{[\mu \nu]} \gamma^{\tau_{1} \tau_{2}}, \quad \partial_{\rho} \gamma^{\mu \nu}=\Gamma_{\rho}^{[\mu \nu]} \tau_{1} \tau_{2} \gamma^{\tau_{1} \tau_{2}}
$$

From eqs- $(26,27)$ one obtains, after performing contractions of the form $\left\langle\gamma^{a b} \gamma_{c d}>=\right.$ constant $\delta_{c d}^{a b}$, the following

$$
\begin{gathered}
\Gamma_{\mathbf{0} \sigma}^{\mu} \delta_{\rho_{1} \rho_{2}}^{\sigma \nu}-\Gamma_{\mathbf{0} \sigma}^{\nu} \delta_{\rho_{1} \rho_{2}}^{\sigma \mu}=\Gamma_{\mathbf{0} \tau_{1} \tau_{2}}^{[\mu \nu]} \delta_{\rho_{1} \rho_{2}}^{\tau_{1} \tau_{2}}=\Gamma_{\mathbf{0} \rho_{1} \rho_{2}}^{[\mu \nu]} \\
\Gamma_{\alpha \sigma \sigma}^{\mu} \delta_{\rho_{1} \rho_{2}}^{\sigma \nu}-\Gamma_{\alpha \sigma}^{\nu} \delta_{\rho_{1} \rho_{2}}^{\sigma \mu}=\Gamma_{\alpha \tau_{1} \tau_{2}}^{[\mu \nu]} \delta_{\rho_{1} \rho_{2}}^{\tau_{1} \tau_{2}}=\Gamma_{\alpha \rho_{1} \rho_{2}}^{[\mu \nu]}
\end{gathered}
$$

Hence from eqs- $(29,30)$ one has an explicit form for $\Gamma_{\mathbf{0} \rho_{1} \rho_{2}}^{[\mu \nu]}, \Gamma_{\alpha \rho_{1} \rho_{2}}^{[\mu \nu]}$ in terms of

$$
\Gamma_{\mathbf{0} \sigma}^{\mu}=\frac{1}{2} g_{\sigma \tau} \partial_{\mathbf{0}} g^{\mu \tau}, \quad \Gamma_{\alpha \sigma}^{\mu}=\frac{1}{2} g_{\sigma \tau} \partial_{\alpha} g^{\mu \tau}
$$

respectively. From the (anti) commutators

$$
\left[\gamma_{m n}, \gamma^{r s}\right]=-8 \delta_{[m}^{[r} \gamma_{n]}^{s]}, \quad\left\{\gamma_{m n}, \gamma^{r s}\right\}=2 \gamma_{m n}^{r s}-4 \delta_{m n}^{r s}
$$

by taking derivatives $\partial / \partial x^{\rho}$ on both sides of the equations one arrives after some algebra, and by lowering indices, to the relations

$$
\begin{gathered}
\Gamma_{\rho[m n]}^{[p q]} g_{[p q][r s]}+\Gamma_{\rho[r s]}^{[p q]} g_{[p q][m n]}=\partial_{\rho}\left(g_{[m n][r s]}\right), \quad g_{[m n][r s]}=g_{[r s][m n]} \\
\Gamma_{\rho[m n]}^{[p q]} \gamma_{p q r s}+\Gamma_{\rho[r s]}^{[p q]} \gamma_{p q m n}=\Gamma_{\rho[m n r s]}{ }^{[a b c d]} \gamma_{a b c d} \\
\Gamma_{\rho[m n]}{ }^{[p q]} \delta_{[p}^{[r} \gamma_{q]}^{s]}+\Gamma_{\rho}^{[r s]}{ }_{[p q]} \delta_{[m}^{[p} \gamma_{n]}^{q]}=\delta_{[m}^{[r} \Gamma_{\rho n] \sigma}^{s]} \gamma_{\tau}^{\sigma}
\end{gathered}
$$

and by taking derivatives with respect to $\partial / \partial x^{\rho_{1} \rho_{2} \ldots \rho_{k}}$, by lowering indices, one arrives at

$$
\Gamma_{\left[\rho_{1} \rho_{2} \ldots \rho_{k}\right][m n]}^{[p q]} g_{[p q][r s]}+\Gamma_{\left[\rho_{1} \rho_{2} \ldots . . \rho_{k}\right][r s]}^{[p q]} g_{[p q][m n]}=\partial_{\rho_{1} \rho_{2} \ldots \rho_{k}}\left(g_{[m n][r s]}\right)
$$




$$
\begin{gathered}
\Gamma_{\left[\rho_{1} \rho_{2} \ldots \rho_{k}\right][m n]}^{[p q]} \gamma_{p q r s}+\Gamma_{\left[\rho_{1} \rho_{2} \ldots . . \rho_{k}\right][r s]}^{[p q]} \gamma_{p q m n}=\Gamma_{\left[\rho_{1} \rho_{2} \ldots . . \rho_{k}\right][m n r s]}{ }^{[a b c d]} \gamma_{a b c d} \\
\Gamma_{\left[\rho_{1} \ldots \rho_{k}\right][m n]}^{[p q]} \delta_{[p}^{[r} \gamma_{q]}^{s]}+\Gamma_{\left[\rho_{1} \ldots \rho_{k}\right]{ }_{[p q]}{ }_{[m}^{[r]} \gamma_{n]}^{[p}}=\delta_{[m}^{[r} \Gamma_{\left.\left[\rho_{1} \ldots \rho_{k}\right] n\right] \sigma}^{s] \tau} \gamma_{\tau}^{\sigma}
\end{gathered}
$$

In this fashion by using the remaining anti (commutators) $\left\{\gamma^{A}, \gamma^{B}\right\},\left[\gamma^{A}, \gamma^{B}\right]$ involving the other Clifford algebra generators (poly-vector basis), one can recursively obtain (define) the $C$-space connections in terms of derivatives of the $C$-space metric $g_{A B}$. One may notice that the expression for the $C$-space connections do not coincide with the LeviCivita-like connections. Since the algebra is very cumbersome a computer Clifford algebra package is necessary. The commutators $\left[\Gamma_{A}, \Gamma_{B}\right]$ for $p q=$ odd one has $[6]$

$$
\begin{gathered}
{\left[\gamma_{b_{1} b_{2} \ldots \ldots b_{p}}, \gamma^{a_{1} a_{2} \ldots \ldots a_{q}}\right]=2 \gamma_{b_{1} b_{2} \ldots \ldots b_{p}}^{a_{1} a_{2} \ldots \ldots a_{q}}-} \\
\frac{2 p ! q !}{2 !(p-2) !(q-2) !} \delta_{\left[b_{1} b_{2}\right.}^{\left[a_{1} a_{2}\right.} \gamma_{\left.b_{3} \ldots \ldots b_{p}\right]}^{\left.a_{3} \ldots a_{q}\right]}+\frac{2 p ! q !}{4 !(p-4) !(q-4) !} \delta_{\left[b_{1} \ldots b_{4}\right.}^{\left[a_{1} \ldots a_{4}\right.} \gamma_{\left.b_{5} \ldots \ldots b_{p}\right]}^{\left.a_{5} \ldots a_{q}\right]}-\ldots \ldots
\end{gathered}
$$

for $p q=$ even one has

$$
\begin{gathered}
{\left[\gamma_{b_{1} b_{2} \ldots . . b_{p}}, \gamma^{a_{1} a_{2} \ldots \ldots a_{q}}\right]=-\frac{(-1)^{p-1} 2 p ! q !}{1 !(p-1) !(q-1) !} \delta_{\left[b_{1}\right.}^{\left[a_{1}\right.} \gamma_{\left.b_{2} b_{3} \ldots \ldots b_{p}\right]}^{\left.a_{2} a_{3} \ldots a_{q}\right]}-} \\
\frac{(-1)^{p-1} 2 p ! q !}{3 !(p-3) !(q-3) !} \delta_{\left[b_{1} \ldots b_{3}\right.}^{\left[a_{1} \ldots a_{3}\right.} \gamma_{\left.b_{4} \ldots \ldots b_{p}\right]}^{\left.a_{4} \ldots a_{q}\right]}+\ldots \ldots
\end{gathered}
$$

The anti-commutators for $p q=$ even are

$$
\begin{gathered}
\left\{\gamma_{b_{1} b_{2} \ldots \ldots b_{p}}, \gamma^{a_{1} a_{2} \ldots \ldots a_{q}}\right\}=2 \gamma_{b_{1} b_{2} \ldots \ldots b_{p}}^{a_{1} a_{2} \ldots \ldots a_{q}}- \\
\frac{2 p ! q !}{2 !(p-2) !(q-2) !} \delta_{\left[b_{1} b_{2}\right.}^{\left[a_{1} a_{2}\right.} \gamma_{\left.b_{3} \ldots \ldots b_{p}\right]}^{\left.a_{3} \ldots a_{q}\right]}+\frac{2 p ! q !}{4 !(p-4) !(q-4) !} \delta_{\left[b_{1} \ldots b_{4}\right.}^{\left[a_{1} \ldots a_{4}\right.} \gamma_{\left.b_{5} \ldots \ldots b_{p}\right]}^{\left.a_{5} \ldots a_{q}\right]}-\ldots \ldots
\end{gathered}
$$

and the anti-commutators for $p q=o d d$ are

$$
\begin{gathered}
\left\{\gamma_{b_{1} b_{2} \ldots . . b_{p}}, \gamma^{a_{1} a_{2} \ldots \ldots a_{q}}\right\}=-\frac{(-1)^{p-1} 2 p ! q !}{1 !(p-1) !(q-1) !} \delta_{\left[b_{1}\right.}^{\left[a_{1}\right.} \gamma_{\left.b_{2} b_{3} \ldots . b_{p}\right]}^{\left.a_{2} a_{3} \ldots a_{q}\right]}- \\
\frac{(-1)^{p-1} 2 p ! q !}{3 !(p-3) !(q-3) !} \delta_{\left[b_{1} \ldots b_{3}\right.}^{a_{1} \ldots a_{3}} \gamma_{\left.b_{4} \ldots . . b_{p}\right]}^{\left.a_{4} \ldots a_{q}\right]}+\ldots \ldots
\end{gathered}
$$

For instance,

$$
\begin{gathered}
{\left[\gamma_{b}, \gamma^{a}\right]=2 \gamma_{b}^{a} ; \quad\left[\gamma_{b_{1} b_{2}}, \gamma^{a_{1} a_{2}}\right]=-8 \delta_{\left[b_{1}\right.}^{\left[a_{1}\right.} \gamma_{\left.b_{2}\right]}^{\left.a_{2}\right]} .} \\
{\left[\gamma_{b_{1} b_{2} b_{3},}, \gamma^{a_{1} a_{2} a_{3}}\right]=2 \gamma_{b_{1} b_{2} b_{3}}^{a_{1} a_{2} a_{3}}-36 \delta_{\left[b_{1} b_{2}\right.}^{\left[a_{1} a_{2}\right.} \gamma_{\left.b_{3}\right]}^{\left.a_{3}\right]} .} \\
\left.\left[\gamma_{b_{1} b_{2} b_{3} b_{4}}, \gamma^{a_{1} a_{2} a_{3} a_{4}}\right]=-32 \delta_{\left[b_{1}\right.}^{\left[a_{1}\right.} \gamma_{\left.b_{2} b_{3} b_{4}\right]}^{\left.a_{2} a_{3} a_{4}\right]}+192 \delta_{\left[b_{1} b_{2} b_{3}\right.}^{\left[a_{1} a_{2} a_{3}\right.} \gamma_{b_{4}}^{\left.a_{4}\right]}\right]
\end{gathered}
$$


etc...

To sum up, the $C$-space connections must be compatible with the Clifford algebra as shown in the above equations and are determined from the algebraic relations imposed by the Clifford algebra itself. In general, $C$-space admits torsion [1]. The $C$-space connections expressions are very different from the Levi-Civita-like connection

$$
\left\{\begin{array}{c}
C \\
A B
\end{array}\right\}=\frac{1}{2} g^{C D}\left(\partial_{A} g_{D B}+\partial_{B} g_{A D}-\partial_{D} g_{A B}\right)
$$

Furthermore, these results should be contrasted with the very restricted ansatz in [1] where one had that $R_{\mu_{1} \mu_{2} \rho_{2}}^{\rho_{1}}=\Gamma_{\left[\mu_{1} \mu_{2}\right] \rho_{2}}^{\rho_{1}}$ when the metric $g_{\mu \nu}$ and connection solely depended on the $x^{\mu}$ coordinates.

The curvatures obey the symmetry relations

$$
\mathbf{R}_{A B C D}=-R_{B A C D}=-R_{A B D C}=R_{C D A B}
$$

and are defined as

$$
\mathbf{R}_{A B C}^{D}=\partial_{A} \Gamma_{B C}^{D}-\partial_{B} \Gamma_{A C}^{D}+\Gamma_{A E}^{D} \Gamma_{B C}^{E}-\Gamma_{B E}^{D} \Gamma_{A C}^{E}
$$

The $C$-space connections are determined in terms of the $C$-space metric $g_{A B}$ by the procedure described above. Some examples of the $C$-space curvatures are

$$
\begin{gathered}
\mathbf{R}_{\left[\mu_{1} \mu_{2} \ldots . . \mu_{2 n}\right] \mathbf{0}\left[\rho_{1} \rho_{2} \ldots . . \rho_{2 n}\right]} \mathbf{0}=\partial_{\mu_{1} \mu_{2} \ldots . . \mu_{2 n}} \Gamma_{\mathbf{0}}^{\mathbf{0}}{ }_{\left[\rho_{1} \rho_{2} \ldots . . \rho_{2 n}\right]}-\partial_{\mathbf{0}} \Gamma_{\left[\mu_{1} \mu_{2} \ldots . \mu_{2 n}\right]\left[\rho_{1} \rho_{2} \ldots . \rho_{2 n}\right]}^{\mathbf{0}}+ \\
\Gamma_{\left[\mu_{1} \mu_{2} \ldots . \mu_{2 n}\right] A}^{\mathbf{0}} \Gamma_{\mathbf{0}\left[\rho_{1} \rho_{2} \ldots \rho_{2 n}\right]}^{A}-\Gamma_{\mathbf{0} A}^{\mathbf{0}{ }_{A}} \Gamma_{\left[\mu_{1} \mu_{2} \ldots . \mu_{2 n}\right]\left[\rho_{1} \rho_{2} \ldots \rho_{2 n}\right]}^{A}
\end{gathered}
$$

The standard Riemann curvature tensor in ordinary spacetime is contained in $C$-space as follows

$$
\begin{gathered}
\mathcal{R}_{\mu_{1} \mu_{2} \rho_{1}}^{\rho_{2}}=\partial_{\mu_{1}} \Gamma_{\mu_{2} \rho_{1}}^{\rho_{2}}-\partial_{\mu_{2}} \Gamma_{\mu_{1} \rho_{1}}^{\rho_{2}}+\Gamma_{\mu_{1} \sigma}^{\rho_{2}} \Gamma_{\mu_{2} \rho_{1}}^{\sigma}-\Gamma_{\mu_{2} \sigma}^{\rho_{2}} \Gamma_{\mu_{1} \rho_{1}}^{\sigma} \subset \\
\mathbf{R}_{\mu_{1} \mu_{2} \rho_{1}}^{\rho_{2}}=\partial_{\mu_{1}} \Gamma_{\mu_{2} \rho_{1}}^{\rho_{2}}-\partial_{\mu_{2}} \Gamma_{\mu_{1} \rho_{1}}^{\rho_{2}}+\Gamma_{\mu_{1} \mathbf{A}}^{\rho_{2}} \Gamma_{\mu_{2} \rho_{1}}^{\mathbf{A}}-\Gamma_{\mu_{2} \mathbf{A}}^{\rho_{2}} \Gamma_{\mu_{1} \rho_{1}}^{\mathbf{A}}
\end{gathered}
$$

due to the contractions involving the poly-vector valued indices $\mathbf{A}$ in eq-(50). There is also the crucial difference that $\mathbf{R}_{\mu_{1} \mu_{2} \rho_{1}}^{\rho_{2}}\left(s, x^{\nu}, x^{\nu_{1} \nu_{2}}, \ldots\right)$ has now an additional dependence on all the $C$-space poly-vector valued coordinates $s, x^{\nu_{1} \nu_{2}}, x^{\nu_{1} \nu_{2} \nu_{3}}, \ldots$ besides the $x^{\nu}$ coordinates.

We shall assume that the mixed-grade $C$-space metric components are zero

$$
g_{0}\left[\nu_{1} \nu_{2} \ldots . . \rho_{i}\right]=0 ; \quad g_{\left[\mu_{1} \mu_{2} \ldots \mu_{i}\right]\left[\nu_{1} \nu_{2} \ldots . . \nu_{j}\right]}=0, \text { when } i \neq j
$$

The non-vanishing $C$-space metric components obeying $g_{A B}=g_{B A}$ are assumed to be of the form

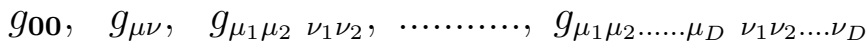


In the most general case the metric does not factorize into antisymmetrized sums of products of the form

$$
\begin{gathered}
g_{\left[\mu_{1} \mu_{2}\right]\left[\nu_{1} \nu_{2}\right]}\left(x^{\mu}\right) \neq g_{\mu_{1} \nu_{1}}\left(x^{\mu}\right) g_{\mu_{2} \nu_{2}}\left(x^{\mu}\right)-g_{\mu_{2} \nu_{1}}\left(x^{\mu}\right) g_{\mu_{1} \nu_{2}}\left(x^{\mu}\right) \\
g_{\left[\mu_{1} \mu_{2} \ldots \mu_{k}\right]\left[\nu_{1} \nu_{2} \ldots \nu_{k}\right]}\left(x^{\mu}\right) \neq \operatorname{det} G_{\mu_{i} \nu_{j}}=\epsilon^{j_{1} j_{2} \ldots \ldots j_{k}} g_{\mu_{1} \nu_{j_{1}}} g_{\mu_{2} \nu_{j_{2}}} \ldots \ldots \ldots g_{\mu_{2} \nu_{j_{k}}}, \quad k=1,2,3, \ldots \ldots D
\end{gathered}
$$

The determinant of $G_{\mu_{i} \nu_{j}}$ can be written as

$$
\operatorname{det}\left(\begin{array}{ccc}
g_{\mu_{1} \nu_{1}}\left(x^{\mu}\right) & \ldots \ldots \ldots \ldots & g_{\mu_{1} \nu_{k}}\left(x^{\mu}\right) \\
g_{\mu_{2} \nu_{1}}\left(x^{\mu}\right) & \ldots \ldots \ldots \ldots & g_{\mu_{2} \nu_{k}}\left(x^{\mu}\right) \\
----------- & ------------ \\
\hline g_{\mu_{k} \nu_{1}}\left(x^{\mu}\right) & \ldots \ldots \ldots \ldots & g_{\mu_{k} \nu_{k}}\left(x^{\mu}\right)
\end{array}\right)
$$

The metric component $g_{\mathbf{0}}$ involving the scalar "directions" in $C$-space of the Clifford polyvectors must also be included. It behaves like a Clifford scalar. The other component $g_{\left[\mu_{1} \mu_{2} \ldots . . \mu_{D}\right]\left[\nu_{1} \nu_{2} \ldots \ldots \nu_{D}\right]}$ involves the pseudo-scalar "directions". The latter scalar and pseudoscalars might bear some connection to the dilaton and axion fields in cosmology and particle physics.

The hyper-determinant of a hyper-matrix [11] can be recast in terms of discriminants [12]. In this fashion one can define the hyper-determinant of $g_{A B}$ as products of the hyper-determinants corresponding to the hyper-matrices

$$
g_{\left[\mu_{1} \mu_{2}\right]\left[\nu_{1} \nu_{2}\right]}, \ldots \ldots . ., g_{\left[\mu_{1} \mu_{2} \ldots \mu_{k}\right]\left[\nu_{1} \nu_{2} \ldots \nu_{k}\right]}, \text { for } 1<k<D
$$

and construct a suitable measure of integration $\mu_{\mathbf{m}}\left(s, x^{\mu}, x^{\mu_{1} \mu_{2}}, \ldots \ldots x^{\mu_{1} \mu_{2} \ldots \mu_{D}}\right)$ in $C$-space which, in turn, would allow us to build the $C$-space version of the Einstein-Hilbert action

$$
\begin{gathered}
\frac{1}{2 \kappa^{2}} \int d s \prod d x^{\mu} \prod d x^{\mu_{1} \mu_{2}} \ldots \ldots d x^{\mu_{1} \mu_{2} \ldots \mu_{D}} \mu_{\mathbf{m}}\left(s, x^{\mu}, x^{\mu_{1} \mu_{2}}, \ldots \ldots x^{\mu_{1} \mu_{2} \ldots \mu_{D}}\right) \mathbf{R}\left(g_{A B}, \Gamma_{A B}^{C}\right)= \\
S=\frac{1}{2 \kappa^{2}} \int[\mathbf{D X}] \mu_{\mathbf{m}}(\mathbf{X}) \mathbf{R}\left(g_{A B}, \Gamma_{A B}^{C}\right)
\end{gathered}
$$

$\kappa^{2}$ is the $C$-space gravitational coupling constant. In ordinary gravity it is set to $8 \pi G_{N}$, with $G_{N}$ being the Newtonian coupling constant. The $C$-space scalar curvature is defined as

$$
\mathbf{R}=\sum_{j=1}^{D} \sum_{k=1}^{D} \mathbf{R}_{\left[\mu_{1} \mu_{2} \ldots \mu_{j}\right]\left[\nu_{1} \nu_{2} \ldots \nu_{k}\right]}^{\left[\mu_{1} \mu_{2} \ldots . \mu_{j}\right]\left[\nu_{1} \nu_{2} \ldots \nu_{k}\right]}+\sum_{j=1}^{D} \mathbf{R}_{\left[\mu_{1} \mu_{2} \ldots \ldots \mu_{j}\right] \mathbf{0}}{ }^{\left[\mu_{1} \mu_{2} \ldots . . \mu_{j}\right] \mathbf{0}}
$$

The measure must obey the relation

$$
[\mathbf{D X}] \mu_{\mathbf{m}}(\mathbf{X})=\left[\mathbf{D X}^{\prime}\right] \mu_{\mathbf{m}}^{\prime}\left(\mathbf{X}^{\prime}\right)
$$

under poly-vector valued coordinate transformations in $C$-space. When the mixed-grade $C$-space metric components are zero, the hyper-determinant of the $C$-space metric $g_{A B}$ factorizes as 
$h \operatorname{det}\left(g_{A B}\right)=g_{00} \operatorname{det}\left(g_{\mu \nu}\right) h \operatorname{det}\left(g_{\mu_{1} \mu_{2} \nu_{1} \nu_{2}}\right)$

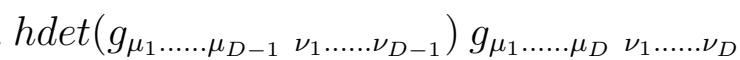

where the hyper-determinant of $g_{\mu \nu}$ coincides with the ordinary determinant of $g_{\mu \nu}$. An immediate question arises, does the Palatini formalism work also in $C$-spaces ? Namely, does a variation with respect to the $C$-space connections $\left(\delta S / \delta \Gamma_{A B}^{C}\right)=0$ yield the same connections as those obtained from the mere structure of the Clifford algebra and depicted above in this section? We leave this question for future work.

\section{On $C$-space and Lanczos-Lovelock Gravity}

The $n$-th order Lanczos-Lovelock curvature tensor is defined as

$$
\mathcal{R}^{(n) \rho_{1} \rho_{1} \rho_{2} \ldots . . . \rho_{2 n}}=\delta_{\mu_{1} \mu_{2} \ldots . . \mu_{2 n}}^{\rho_{1} \rho_{2} \ldots \rho_{2 n}} \delta_{\mu_{1} \tau_{2} \ldots . \tau_{2 n}}^{\nu_{1} \nu_{2} \ldots \nu_{2 n}} \mathcal{R}_{\nu_{1} \nu_{2}}^{\tau_{1} \tau_{2}} \mathcal{R}_{\nu_{3} \nu_{4}}^{\tau_{3} \tau_{4}} \ldots \ldots . . \mathcal{R}_{\nu_{2 n-1} \nu_{2 n}}^{\tau_{2 n-1} \tau_{2 n}}
$$

the $n$-th order Lovelock curvature scalar is

$$
\mathcal{R}^{(n)}=\delta_{\tau_{1} \tau_{2} \ldots \tau_{2 n}}^{\nu_{1} \nu_{2} \ldots \nu_{2 n}} \mathcal{R}_{\nu_{1} \nu_{2}}^{\tau_{1} \tau_{2}} \mathcal{R}_{\nu_{3} \nu_{4}}^{\tau_{3} \tau_{4}} \ldots \ldots . . \mathcal{R}_{\nu_{2 n-1} \nu_{2 n}}^{\tau_{2 n-1} \tau_{2 n}}
$$

the Riemann curvature tensor obeys the relations

$$
\mathcal{R}_{\mu_{1} \mu_{2} \rho_{1} \rho_{2}}=\mathcal{R}_{\rho_{1} \rho_{2} \mu_{1} \mu_{2}}=-\mathcal{R}_{\mu_{2} \mu_{1} \rho_{1} \rho_{2}}=-\mathcal{R}_{\mu_{1} \mu_{2} \rho_{2} \rho_{1}}
$$

After lowering the indices for the $n$-order Lanczos-Lovelock curvature tensors gives the symmetry relation

$$
\mathcal{R}_{\rho_{1} \rho_{2} \ldots . . \rho_{2 n} \mu_{1} \mu_{2} \ldots \mu_{2 n}}^{(n)}=\mathcal{R}_{\mu_{1} \mu_{2} \ldots . . \mu_{2 n} \rho_{1} \rho_{2} \ldots \rho_{2 n}}^{(n)}
$$

the above curvature tensors are antisymmetric under the exchange of any of the $\mu(\nu)$ indices, respectively, in addition to obeying the (generalized) Bianchi identities [7], [8].

The Lanczos-Lovelock Lagrangian density is

$$
\mathcal{L}=\sqrt{g} \sum_{n=0}^{\left[\frac{D}{2}\right]} c_{n} \mathcal{L}_{n}, \quad \mathcal{L}_{n}=\frac{1}{2^{n}} \mathcal{R}^{(n)}
$$

where $c_{n}$ are arbitrary coefficients; the first term corresponds to the cosmological constant. The integer part is $\left[\frac{D}{2}\right]=\frac{D}{2}$ when $D=$ even, and $\frac{D-1}{2}$ when $D=$ odd. The general Lanczos-Lovelock theory in $D$ spacetime dimensions is given by the action

$$
S=\int d^{D} x \sqrt{|g|} \sum_{n=0}^{\left[\frac{D}{2}\right]} c_{n} \mathcal{L}_{n}
$$

One of the key properties of Lanczos-Lovelock gravity is that the field equations do not contain higher derivatives of the metric tensor beyond the second order due to the fact 
that the action does not contain derivatives of the curvature, see [7], [8] and references therein.

In this section we will study the very special case when the $C$-space metric $g_{A B}$ solely depends on the coordinates $x^{\mu}$ and explore its relationship to Lanczos-Lovelock gravity in this special case. The $C$-space version of Einstein's equations with a cosmological constant is

$$
\mathbf{R}_{A B}-\frac{1}{2} g_{A B} \mathbf{R}+\Lambda g_{A B}=0
$$

The $C$-space curvature scalar is given by the sum of the contractions

$$
\mathbf{R}=\sum_{j=1}^{D} \sum_{k=1}^{D} \mathbf{R}_{\left[\mu_{1} \mu_{2} \ldots \mu_{j}\right]\left[\nu_{1} \nu_{2} \ldots \nu_{k}\right]}^{\left[\mu_{1} \mu_{2} \ldots \mu_{j}\right]\left[\nu_{1} \nu_{2} \ldots \nu_{k}\right]}+\sum_{j=1}^{D} \mathbf{R}_{\left[\mu_{1} \mu_{2} \ldots . \mu_{j}\right] \mathbf{0}}\left[\mu_{1} \mu_{2} \ldots . \mu_{j}\right] \mathbf{0}
$$

and the $C$-space Ricci-like curvature is

$$
\mathbf{R}_{A}^{B}=\sum_{j=1}^{D} \mathbf{R}_{A\left[\nu_{1} \nu_{2} \ldots \nu_{j}\right]}^{B\left[\nu_{1} \nu_{2} \ldots \nu_{j}\right]}+\mathbf{R}_{A}^{B} \mathbf{0} 0
$$

The $C$-space version of Einstein's equations (66) determine the $C$-space metric $g_{A B}$ when the $C$-space connections are given in terms of derivatives of $g_{A B}$ as shown in section 1 .

One may write the Lanczos-Lovelock gravitational equations in the form [7], [8]

$$
\mathcal{G}_{\mu \nu}=\sum_{n=0}^{\left[\frac{D}{2}\right]} c_{n}\left(n \mathcal{R}_{\mu \nu}^{(n)}-\frac{1}{2} g_{\mu \nu} \mathcal{R}^{(n)}\right)=0
$$

which are more suitable to compare with the $C$-space gravity equations (66) .

The embedding of the Lanczos-Lovelock gravitational equations into the $C$-space gravitational equations requires

$$
\mathcal{G}_{\sigma}^{\rho}=0 \leftrightarrow \mathbf{R}_{\sigma}^{\rho}-\frac{1}{2} \delta_{\sigma}^{\rho} \mathbf{R}+\Lambda \delta_{\sigma}^{\rho}=0
$$

where the $C$-space Ricci-like curvature $\mathbf{R}_{\sigma}{ }^{\rho}$ is

$$
\mathbf{R}_{\sigma}^{\rho}=\sum_{j=1}^{D} \mathbf{R}_{\left[\mu_{1} \mu_{2} \ldots . \mu_{j}\right] \sigma}^{\left[\mu_{1} \mu_{2} \ldots \mu_{j}\right] \rho}+\mathbf{R}_{\sigma \mathbf{0}}{ }^{\rho \mathbf{0}}
$$

and the Ricci-like scalar is given by eq-(67) .

The latter equations are just members of the more general $C$-space field equations given by eq-(66) involving all the poly-vector valued indices. We should emphasize that in order to match units one has to include suitable powers of the Planck length scale $L_{P}$ in the summands in all of our equations. By recurring to eqs-(66) one finds that we can embed the Lanczos-Lovelock gravitational equations (69) into the $C$-space gravity equations if the following conditions on the $C$-space curvatures are satisfied 


$$
\begin{aligned}
& \sum_{n=1}^{\left[\frac{D}{2}\right]} c_{n} n \delta_{\left[\nu_{1}\right.}^{\rho} \delta_{\left.\sigma \nu_{2} \nu_{3} \ldots \nu_{2 n}\right]}^{\mu_{1} \mu_{2} \ldots . \mu_{2 n}} \mathcal{R}_{\mu_{1} \mu_{2} \ldots . . \mu_{2 n}}^{(n) \quad \nu_{1} \nu_{2} \ldots . \nu_{2 n} n}= \\
& \sum_{j=1}^{D} \mathbf{R}_{\mu_{1} \mu_{2} \ldots \ldots \mu_{j} \sigma}^{\mu_{1} \mu_{2} \ldots . \mu_{j} \rho} \rho+\mathbf{R}_{\mathbf{0}}{ }_{\sigma}^{\mathbf{0} \rho}
\end{aligned}
$$

and

$$
\begin{aligned}
& \sum_{n=1}^{\left[\frac{D}{2}\right]} c_{n} \delta_{\nu_{1} \nu_{2} \ldots \nu_{2 n}}^{\mu_{1} \mu_{2} \ldots . \mu_{2 n}} \mathcal{R}_{\mu_{1} \mu_{2} \ldots . . \mu_{2 n}}^{(n) \quad \nu_{1} \nu_{2} \ldots \ldots \nu_{2 n}}= \\
& \sum_{j=1}^{D} \sum_{k=1}^{D} \mathbf{R}_{\mu_{1} \mu_{2} \ldots \ldots \mu_{j} \nu_{1} \nu_{2} \ldots \ldots \nu_{k}}^{\mu_{1} \mu_{2} \ldots \ldots \mu_{j} \nu_{1} \nu_{2} \ldots \ldots \nu_{k}}+\sum_{j=1}^{D} \mathbf{R}_{\mu_{1} \mu_{2} \ldots \ldots \mu_{j} \mathbf{0}}^{\mu_{1} \mu_{2} \ldots \ldots \mu_{j}} \mathbf{0}
\end{aligned}
$$

One should notice the key factors of $n c_{n}$ in eq-(72) compared with the $c_{n}$ factors in eq- $(73)$. The $n=0$ term in (69) corresponds to the cosmological constant

$$
c_{o} \mathcal{R}^{(0)}=-2 \Lambda \Rightarrow-\frac{1}{2} c_{o} \mathcal{R}^{(0)}=\Lambda
$$

The above embedding conditions $(72,73)$ can be simplified considerable if one has the following vanishing traces

$$
\begin{gathered}
\mathbf{R}_{\nu_{1} \nu_{2} \ldots \ldots \nu_{2 n-1} \mathbf{0}}^{\nu_{1} \nu_{2} \ldots \nu_{2 n-1} \mathbf{0}}=0, \quad \mathbf{R}_{\nu_{1} \nu_{2} \ldots \ldots \nu_{2 n-1} \rho_{1} \rho_{2} \ldots \ldots \rho_{k}}^{\nu_{1} \nu_{2} \ldots \ldots \nu_{2 n-1}} \rho_{\rho_{2} \rho_{2} \ldots \ldots}=0 \\
\mathbf{R}_{\nu_{1} \nu_{2} \ldots \ldots \nu_{2 n-1} \sigma}^{\nu_{1} \nu_{2} \ldots \ldots \nu_{2 n-1} \rho}=0
\end{gathered}
$$

However the introduction of these vanishing traces will lead to an over-determined system of equations, in conjunction with the $C$-space field equations. As it happens with an overdetermined system of equations one is hard pressed to find nontrivial solutions $R_{A B C D} \neq 0$. For this reason we shall refrain from introducing additional equations like (75).

One may begin by solving the Lanczos-Lovelock (LL) equations (69) which determine the ordinary metric components $g_{\mu \nu}\left(x^{\rho}\right)$, the connection $\Gamma_{\mu \nu}^{\rho}\left(x^{\mu}\right)$ and the LL curvature tensor $\mathcal{R}_{\mu_{1} \mu_{2} \ldots \ldots \mu_{2 n}}^{(n) \rho_{1} \rho_{2} \ldots \rho_{2 n}}\left(x^{\mu}\right)$. Let us look for the maximally symmetric constant curvature vacua solutions to Lovelock gravity, like de Sitter and Anti de Sitter spaces in ordinary Einstein gravity. The Riemann tensor in the latter case is

$$
\begin{gathered}
R_{\mu_{1} \mu_{2} \rho_{1} \rho_{2}}=\frac{2 \Lambda}{(D-1)(D-2)}\left(g_{\mu_{1} \rho_{1}}\left(x^{\mu}\right) g_{\mu_{2} \rho_{2}}\left(x^{\mu}\right)-g_{\mu_{2} \rho_{1}}\left(x^{\mu}\right) g_{\mu_{1} \rho_{2}}\left(x^{\mu}\right)\right) \Rightarrow \\
R_{\mu_{1} \mu_{2}}^{\rho_{1} \rho_{2}}=\left[\frac{2 \Lambda}{(D-1)(D-2)}\right] \delta_{\mu_{1} \mu_{2}}^{\rho_{1} \rho_{2}} \Rightarrow R=\frac{2 D}{D-2} \Lambda
\end{gathered}
$$

so that the $n$-th order constant Lanczos-Lovelock (LL) curvature tensor is

$$
R_{\mu_{1} \mu_{2} \ldots . . \mu_{2 n}}^{(n) \rho_{1} \rho_{2} \ldots \rho_{2 n}}=\left[\frac{2 \Lambda}{(D-1)(D-2)}\right]^{n} \delta_{\mu_{1} \mu_{2} \ldots \ldots \mu_{2 n}}^{\rho_{1} \rho_{2} \ldots \rho_{2 n}}
$$


One must still check that the curvatures (77) are solutions to the Lanczos-Lovelock gravitational equations. In [9] the authors have shown that black-brane (black-string) solutions to Lanczos-Lovelock gravity theories in higher dimensions $(D>4)$ including higher curvature terms may, in fact, be simply constructed, but only within a certain class of Lanczos-Lovelock theories. This class of theories had the following property. Assume that $L_{r}$ is the highest order term in the Lagrangian, i.e. that the coefficients $c_{n}$ vanish for $n>r$. Depending on the values of the nonzero coefficients in the Lagrangian, it then turns out that the theory may have up to $r$ distinct constant curvature vacuum solutions [9]. The different values that the constant curvature may take are the roots of a $r$-th order polynomial. There will, of course, generally be $r$ roots, but only real values of the curvature are considered to be physical. The coefficients in the Lanczos-Lovelock Lagrangian may be tuned such that there are $r$ real roots and that all these roots coincide. The theory then has a (locally) unique constant curvature vacuum solution. The authors [9] referred to these as LUV theories - standing for Lovelock-Unique-Vacuum. Those LUV theories are the ones which have simple black-brane solutions.

For the purposes of studying LUV theories, the authors [9] found it very useful to rewrite the LL equations of motion $\mathcal{G}_{a b}=0$ in an alternative form which was very useful as we shall see below,

$$
\mathcal{G}^{a}{ }_{b}=\alpha_{0} \delta_{b \nu_{1} \nu_{2} \ldots \nu_{2 r}}^{a \mu_{1} \mu_{2} \ldots \mu_{2 r}}\left(R_{\mu_{1} \mu_{2}}^{\nu_{1} \nu_{2}}+\alpha_{1} \delta_{\mu_{1} \mu_{2}}^{\nu_{1} \nu_{2}}\right) \cdots\left(R_{\mu_{2 r-1} \mu_{2 r}}^{\nu_{2 r-1} \nu_{2 r}}+\alpha_{r} \delta_{\mu_{2 r-1} \nu_{2 r}}^{\nu_{2 r-1} \nu_{2 r}}\right) .
$$

The original form of the equations of motion can then be recovered through repeated applications of the identity

$$
\delta_{b_{1} \ldots b_{p}}^{a_{1} \ldots a_{p}} \delta_{a_{p-1} a_{p}}^{b_{p-1} b_{p}}=2(D-(p-1))(D-(p-2)) \delta_{b_{1} \ldots b_{p-2}}^{a_{1} \ldots a_{p-2}}
$$

The coefficients $c_{n}$ are given by sums of products of the parameters $\alpha_{n}$. The precise relation is given in reference [10]. Inverting this relation to get the $\alpha_{n}$ 's in terms of the $c_{n}$ 's requires solving a polynomial equation of order $r$. Hence the $\alpha_{n}$ 's are generally complex parameters.

When each one of the factors in eq-(78) becomes zero

$$
R_{\mu_{i} \mu_{i+1}}^{\nu_{i} \nu_{i+1}}+\alpha_{i} \delta_{\mu_{i} \mu_{i+1}}^{\nu_{i} \nu_{i+1}}=0 \Rightarrow R_{\mu_{i} \mu_{i+1}}^{\nu_{i} \nu_{i+1}}=-\alpha_{i} \delta_{\mu_{i} \mu_{i+1}}^{\nu_{i} \nu_{i+1}}, \quad i=1,2,3, \ldots ., r
$$

one has then several different possible values for the constant curvature vacuum solutions. The LUV theories discussed above result from setting all the parameters $\alpha_{n}$ with $n=$ $1, \ldots r$ equal to a common value $\alpha$ and that is related to the cosmological constant $\Lambda$. There is then, at least locally, a unique constant curvature vacuum. If we further set $\alpha=0$, we get a pure Lovelock theory with Lagrangian $\mathcal{L}=\alpha_{0} \mathcal{L}_{r}$, which has flat spacetime as its unique constant curvature vacuum.

Having found the values for $c_{n}$ which yield the LUV solutions, one still has to verify that the special case solutions for $g_{\mathbf{0 0}}\left(x^{\mu}\right)$ and $g_{\mu_{1} \mu_{2} \ldots \mu_{k} \nu_{1} \nu_{2} \ldots \nu_{k}}\left(x^{\mu}\right)$ solve the $C$-space gravitational equations (66) when the ordinary metric $g_{\mu \nu}\left(x^{\mu}\right)$ belongs to the LUV class of solutions to the Lanczos-Lovelock gravitational equations $(69,78)$ discussed above. For particular (black-brane) solutions for $g_{\mu \nu}$ we refer to [9] and references therein. 
For the constant-curvature vacuum solutions case, the left hand side of the above embedding conditions $(72,73)$ become

$$
\sum_{n=1}^{\left[\frac{D}{2}\right]} c_{n} n \delta_{\sigma}^{\rho}\left[\frac{2 \Lambda}{(D-1)(D-2)}\right]^{n}\left[\frac{D !}{(D-(2 n-1)) !}\right]
$$

and

$$
\sum_{n=1}^{\left[\frac{D}{2}\right]} c_{n}\left[\frac{2 \Lambda}{(D-1)(D-2)}\right]^{n}\left[\frac{D !}{(D-2 n) !}\right]
$$

As usual, on must adjust units in eqs- $(81,82)$ by taking into account that the dimensionful coefficients $c_{n}$ are given in terms of powers of $L_{P}$ so that the Lanczos-Lovelock action in $D$-dimensions is dimensionless. Having determined that the dimensions of the LanczosLovelock Lagrangian density is $(\text { length })^{-D}$ this fixes the appropriate powers of $L_{P}$ which must appear in the right hand side and left hand side of eqs- $(72,73,81,82)$.

The right hand side of the embedding conditions $(72,73)$, when the left hand side is given by eqs- $(81,82)$, respectively, must contain expressions of the form

$$
\begin{aligned}
\sum_{j=1}^{D} \mathbf{R}_{\mu_{1} \mu_{2} \ldots \ldots \mu_{j} \sigma}^{\mu_{1} \mu_{2} \ldots \ldots \mu_{j} \rho} & =\sum_{j=1}^{D} \delta_{\sigma}^{\rho} a_{j}(D)\left[\frac{2 \Lambda}{(D-1)(D-2)}\right]^{\frac{j+1}{2}} \\
\mathbf{R}_{\mathbf{0} \sigma}^{\mathbf{0} \rho}= & a_{0}(D) \delta_{\sigma}^{\rho}\left[\frac{2 \Lambda}{(D-1)(D-2)}\right]^{\frac{1}{2}}
\end{aligned}
$$

and

$$
\begin{aligned}
\sum_{j=1}^{D} \sum_{k=1}^{D} \mathbf{R}_{\mu_{1} \mu_{2} \ldots \ldots \mu_{j} \nu_{1} \nu_{2} \ldots . . \nu_{k}}^{\mu_{1} \mu_{2} \ldots \ldots \nu_{j} \nu_{1} \nu_{2} \ldots \ldots \nu_{k}} & =\sum_{j=1}^{D} \sum_{k=1}^{D} b_{j k}(D)\left[\frac{2 \Lambda}{(D-1)(D-2)}\right]^{\frac{j+k}{2}} \\
\sum_{j=1}^{D} \mathbf{R}_{\mu_{1} \mu_{2} \ldots . . \mu_{j} \mathbf{0}}^{\mu_{1} \mu_{2} \ldots \ldots \mu_{j}} \mathbf{0} & =\sum_{j=1}^{D} b_{j}(D)\left[\frac{2 \Lambda}{(D-1)(D-2)}\right]^{\frac{j}{2}}
\end{aligned}
$$

where $a_{j}(D), a_{o}(D), b_{j k}(D), b_{j}(D)$ are suitable $D$-dependent dimensionful coefficients in powers of the Planck scale and which are constrained by the values of the $c_{n}$ coefficients which are known for the LUV solutions. It is beyond the scope of this work to find nontrivial solutions to the embedding conditions $(72,73)$ associated with the specific expressions in eqs-(81-84). This is a very challenging problem.

A plausible guide of how to solve such problem might be by recasting the problem in terms of generalized gauge field theories, like generalized Maxwell and Yang-Mills theories. The antisymmetry property of ordinary differential forms involving the coordinates $d x^{\mu} \wedge d x^{\nu}=-d x^{\nu} \wedge d x^{\mu}$ becomes now for bivector coordinate differentials $d x^{\mu_{1} \mu_{2}} \wedge d x^{\rho_{1} \rho_{2}}=d x^{\rho_{1} \rho_{2}} \wedge d x^{\mu_{1} \mu_{2}}$. Similarly one has

$$
d x^{\mu_{1} \mu_{2} \ldots \mu_{2 n}} \wedge d x^{\rho_{1} \rho_{2} \ldots \rho_{2 n}}=d x^{\rho_{1} \rho_{2} \ldots \ldots \rho_{2 n}} \wedge d x^{\mu_{1} \mu_{2} \ldots \ldots \mu_{2 n}}
$$

and

$$
d x^{\mu_{1} \mu_{2} \ldots \mu_{2 n-1}} \wedge d x^{\rho_{1} \rho_{2} \ldots \rho_{2 n-1}}=-d x^{\rho_{1} \rho_{2} \ldots . \rho_{2 n-1}} \wedge d x^{\mu_{1} \mu_{2} \ldots \ldots \mu_{2 n-1}}
$$


One may rewrite our expressions in the language of poly-differential forms, for instance

$$
\begin{aligned}
\mathbf{F}^{(2)} & =\mathbf{d} \mathbf{A}, \quad \mathbf{d}=d x^{\mu_{1} \mu_{2}} \frac{\partial}{\partial x^{\mu_{1} \mu_{2}}}, \quad \mathbf{A}=A_{\rho_{1} \rho_{2}} d x^{\rho_{1} \rho_{2}} \\
\mathbf{F}^{(2)} & =\left(\partial_{\mu_{1} \mu_{2}} A_{\rho_{1} \rho_{2}}+\partial_{\rho_{1} \rho_{2}} A_{\mu_{1} \mu_{2}}\right) d x^{\mu_{1} \mu_{2}} \wedge d x^{\rho_{1} \rho_{2}}
\end{aligned}
$$

one should notice the + sign in eq-(88) due to the properties of eq-(85) .

$$
\mathbf{F}^{(2 n)}=\mathbf{F}^{(2)} \wedge \mathbf{F}^{(2)} \wedge \ldots \ldots \wedge \mathbf{F}^{(2)}(n \text { factor } s)
$$

Therefore, from the functional form of the Lanczos-Lovelock curvatures one can infer the correspondence

$$
\mathbf{F}^{(2)} \leftrightarrow \mathcal{R}_{\mu_{1} \mu_{2}}^{\rho_{1} \rho_{2}}, \quad \mathbf{F}^{(2 n)} \leftrightarrow \mathcal{R}_{\mu_{1} \mu_{2} \ldots . . \mu_{2 n}}^{(n)} \rho_{1} \rho_{2} \ldots \rho_{2 n}
$$

which might aid us in finding nontrivial solutions to eqs-(81-84) when we replace $\mathbf{F}=\mathbf{d} \mathbf{A}$ for the nonabelian version $\mathbf{F}=(\mathbf{d}+\mathbf{A}) \wedge \mathbf{A}$. Furthermore, one still has to use the remaining of the $C$-space gravitational equations (66) for the other poly-vector valued components $g_{A B}$ of the metric. This needs to be solved before one can ascertain that nontrivial solutions of the Lanczos-Lovelock gravitational equations (69) solve the $C$ space gravitational equations (66) in a very special case when $g_{A B}$ only depends on $x^{\mu}$. Perhaps in this particular case the solutions for the $C$-space metric components admit a factorization into anti-symmetrized sums of products of $g_{\mu \nu}$. The plausible relation to extended gravitational theories based on $f(R), f\left(R_{\mu \nu}\right) \ldots$ actions for polynomial-valued functions, and which obviate the need for dark matter, warrants also further investigation [13].

To finalize we should add that Polyvector-valued gauge field theories in noncommutative Clifford spaces were presented in [14] where we found that the study of $n$-ary algebras leads to interesting relationships among the $\mathbf{n}$-ary commutators of noncommuting spacetime coordinates $\left[X^{\mu_{1}}, X^{\mu_{2}}, \ldots \ldots, X^{\mu_{n}}\right]$ with the poly-vector valued coordinates $X^{\mu_{1} \mu_{2} \ldots . . \mu_{n}}$ in noncommutative Clifford spaces. It was given by $\left[X^{\mu_{1}}, X^{\mu_{2}}, \ldots \ldots, X^{\mu_{n}}\right]=n ! X^{\mu_{1} \mu_{2} \ldots \ldots \mu_{n}}$. These findings will be relevant for the quantization program.

\section{Acknowledgements}

We are indebted to M. Bowers for assistance.

\section{References}

[1] C. Castro and M. Pavsic, Progress in Physics vol 1 (April 2005) 31. Phys. Letts B 559 (2003) 74. Int. J. Theor. Phys 42 (2003) 1693.

[2] D. Hestenes, "Spacetime Algebra" Gordon and Breach, New York, 1996.

D. Hestenes and G. Sobcyk, Clifford Algebra to Geometric Calculus (D. Reidel Publishing Company, Dordrecht, 1984). 
[3] M. Pavsic, The Landscape of Theoretical Physics : A Global View, from point particles to the brane world and beyond, in search of a Unifying Principle, (Fundamental Theories of Physics, vol. 19, Kluwer Academic Publishers, Dordrecht, Boston, London, 2001).

[4] I. R. Porteous, Clifford Algebras and the Classical Groups (Cambridge University Press, 1995).

[5] W. Baylis, Electrodynamics, A Modern Geometric Approach, Boston (Birkhauser, 1999).

[6] K. Becker, M. Becker and J. Schwarz, String Theory and M-Theory : A Modern Introduction (Cambridge University Press, 2007, pp. 543-545).

[7] C. Lanczos, Annals Math. 39, 842 (1938). D. Lovelock, J. Math. Phys 12 (1971) 498.

[8] D. Kastor, "The Riemann-Lovelock Curvature Tensor" arXiv : 1202.5287.

N. Dadhich and S. Jhingan, "The Lovelock gravity in the critical spacetime dimension" arXiv : 1205.4575.

T. Sisman, I. Gullu and B. Tekin, "Spectra, vacua and the unitarity of Lovelock gravity in D-dimensional AdS spacetimes" arXiv : 1204.3814.

[9] D. Kastor and R. Mann, "On black strings and branes in Lovelock gravity" hepth/0603168.

[10] J. Crisostomo, R. Troncoso and J. Zanelli, Phys. Rev. D 62, 084013 (2000) [arXiv:hep-th/0003271].

[11] A. Cayley, Cambridge Math. J. 4 (1845) 193.

[12] I. Gelfand, M. Kapranov and A. Zelevinsky, Discriminants, Resultants and Determinants (Birkhauser 1994).

[13] S. Capozziello and M. De Laurentis, "Extended Theories of Gravity" [arXiv:1108.6266] (to appear in Physics Reports).

[14] C. Castro, J. Phys. A : Math. Theor. 43 (2010) 365201. 